\title{
Correlation between Serum Krebs von den Lungen-6 Levels with Forced Vital Capacity and Modified Rodnan Skin Score of Patients with Restrictive Lung Disease in Diffuse-Type Systemic Sclerosis
}

\author{
Herlina Yani ${ }^{1}$, Sumartini Dewi ${ }^{1}$, Andri Reza Rahmadi ${ }^{1}$ \\ ${ }^{1}$ Rheumatology Division, Internal Medicine Department, Faculty of Medicine Universitas Padjadjaran /Hasan Sadikin General \\ Hospital, Bandung Indonesia
}

\begin{abstract}
A R T I C L E I N F O
Keywords:

Diffuse type systemic sclerosis

Forced Vital Capacity

KL-6

mRSS

Restrictive lung disease

Corresponding author:

E-mail address: yani_herlina@yahoo.co.id

All authors have reviewed and approved the final version of the manuscript.
\end{abstract}

https://doi.org/10.37275/IJR.v11i2.133

\section{A B S T R A C T}

Background Pulmonary fibrosis / intersitial lung disease (ILD) in systemic sclerosis (SSc) is a complicated restrictive pulmonary disease and the leading cause of disease-related mortality. Progressive skin fibrosis in diffuse-type SSc (dSSc) is associated with decreased forced vital capacity (FVC). Modified Rodnan Skin Score (mRSS) examination is used as a parameter to assess skin fibrosis, while high-resolution computed tomography (HRCT) and pulmonary function tests (PFTs) are used to assess pulmonary fibrosis. The HRCT test remains as the gold standard in diagnosing ILD. However, it costs a lot and is not available in all healthcare facilities. Krebs Von den Lungen-6 (KL-6) is a biomarker to evaluate pulmonary fibrosis. The aim of this study was to analyze the correlation of serum KL-6 levels with FVC and mRSS value of patients with restrictive lung disease in dSSc. Methods This was a cross-sectional study that used primary data from dSSc patients who visited rheumatology outpatient clinic in Hasan Sadikin Hospital Bandung, Indonesia, during the period of June-July 2019. History taking, physical examination, mRSS, spirometry, and serum KL- 6 levels were performed. Data were analyzed using the Rank Spearman correlation test. Results There were 27 subjects with the mean age of $42 \pm 12$ years. Based on FVC (\%) restrictive lung disease criteria, the majority of subjects $(74.1 \%)$ had severe restrictive lung disease and the rest of all subjects $(25.9 \%)$ were non severe restrictive lung disease. Serum KL-6 levels ranged from 0.545 to $8.138 \mathrm{ng} / \mathrm{ml}$. The results showed that there was no correlation between serum KL-6 levels and FVC values $(r=$ $-0.118, \mathrm{p}=0.279)$ and $\mathrm{mRSS}(\mathrm{r}=0.101, \mathrm{p}=0.312)$.

Conclusion There is no correlation between serum KL-6 levels with FVC and mRSS value of patient with restritive lung disease in diffuse type systemic sclerosis.

\section{Introduction}

Systemic sclerosis (SSc) is a chronic and progressive autoimmune disease hallmarked by the distinctive triad of microvascular damage, dysregulation of innate and adaptive immunity, and most importantly, generalized fibrosis in multiple organs. ${ }^{1}$ Interstitial lung disease (ILD) is the most 
common pulmonary manifestation of SSc, affecting about $40 \%$ of patients, and by far the leading cause of morbidity and mortality. ${ }^{2,3}$ Patients with SSc who develop ILD have increased mortality by 2.9 -fold when compared with SSc patients without ILD. ${ }^{4}$ Furthermore, based on autopsy series performed in SSc patients, pulmonary fibrosis was found in 90 percent of cases. ${ }^{6}$ For those particular reasons, evaluation of ILD in SSc patients is necessary.

High Resolution Computed Tomography (HRCT) scanning remains as the standard and the most sensitive method in diagnosing ILD.7,8 However, HRCT is not available in all healthcare facilities and also not affordable for most patients. 9 Hence, pulmonary function test (PFT) is commonly used to screen and evaluate long term pulmonary function. Decreased forced vital capacity (FVC) determines the severity level of restrictive lung disorder.

More extensive skin thickening corresponds with more severe visceral organ manifestation. ${ }^{10}$ Modified Rodnan skin score (mRSS) is considered as the most appropriate tools in estimating skin fibrosis in SSc. ${ }^{10}$ The progressivity of skin fibrosis in diffuse type SSc (dSSc), which is more severe compared with limited type SSc (1SSc), is associated with decline in lung function and worse survival. ${ }^{11}$ It is a challenge for clinicians to identify ILD in earlier stage of SSc. Therefore, biomarker that could easily detect and significantly portraying lung fibrosis activity is necessary.

Recent studies found that Krebs von den Lungen6 (KL-6) is expressed by alveolar pneumocyte and increased in serum when there is fibrogenesis activity in the lungs. ${ }^{12,13}$ However, the correlation between KL- 6 with FVC and mRSS is still controversial. This study is the pilot study in Indonesia to analyze the correlation between serum KL-6 levels with FVC and mRSS value of patients with restrictive lung disease in dSSc.

\section{Methods}

This was a cross-sectional study conducted in rheumatology outpatient clinic of Hasan Sadikin Hospital Bandung, Indonesia from June until July 2019. The subjects were dSSc patients with restrictive lung disorder confirmed by spirometry test evaluated by the pulmonologist in Hasan Sadikin Hospital Bandung. Decreased FVC of $<80 \%$ and increased FEV1/FVC ratio $>0.7$ were defined as restrictive lung disorder. The patients whom spirometry results met these criteria were included as the subjects in this study. The exclusion criteria were as followed: 1) other autoimmune diseases that can cause restrictive lung disorder (i.e. rheumatoid arthritis, systemic lupus erythematosus, ankylosing spondylitis, and Sjogren syndrome), 2) restrictive lung disorder caused by the other diseases than SSc, 3) malignancy, 4) pneumonia, TB pulmonary, asthma bronchiale, and bronchiectasis, 5) hepatitis, cholecystitis, and pancreatitis, 6) pregnancy and lactation. These exclusion criteria were based on previous studies that reported an elevated level of KL6 on those diseases and pregnancy that could lead to bias in this study. ${ }^{13}$

History taking, physical examination, and other supporting examination written in subjects' medical record were evaluated to exclude the subject. This study collected primary data consisting of serum KL6 from subjects, their skin fibrosis condition assessed by mRSS, and FVC value measured by spirometry test. KL-6 / human mucin-1 (Human MUC-1, ng/mL) serum were measured from 5 milliliters of venous blood sample with ELISA sandwich method.

Baseline characteristics, including age, gender, duration of disease, clinical manifestation, and treatment history received by subjects, were also collected during the visit. Statistical analysis used in this study were Spearman correlation test. This study also has been approved by Hasan Sadikin General Hospital Ethics and Research Committee.

\section{Results}

There were 27 subjects included in this study and all of them were female with the mean age of 42 years old. Characteristics of the subjects were shown in Table 1. Median value of KL-6 serum was 2.999 $\mathrm{ng} / \mathrm{mL}$ with the range of $0.545-8.138 \mathrm{ng} / \mathrm{mL}$. Median score of mRSS of the subjects was 23 (range: $5-45$ ) and mean of FVC percentage was $52 \%$. There were 21 subjects who had received methotrexate as disease-modifying antirheumatic drugs (DMARDs) before, 3 subjects who had DMARDs other than methotrexate (azathioprine and cyclophosphamide), and 3 subjects who were DMARDs naive.

This study showed a very weak negative correlation between KL-6 serum and FVC value. However it was not significant $(r=-0,118, p=0,279)$ as shown in Table 2 and Figure 1. On the other hand, there was a weak positive correlation between KL-6 serum and mRSS score which was not significant either $(r=0,101, p=0,312$, Table 3$)$ 
Table 1. Characteristics of Subjects

\begin{tabular}{|c|c|}
\hline Variable & $\mathbf{n}=\mathbf{2 7}$ \\
\hline Age (years), mean $\pm \mathrm{SD}$ & $42 \pm 12$ \\
\hline \multicolumn{2}{|l|}{ Gender, n (\%) } \\
\hline Female & 27 (100) \\
\hline Male & $0(0)$ \\
\hline Duration of disease (years), median (range) & $3(1-23)$ \\
\hline \multicolumn{2}{|l|}{ Treatment history, n (\%) } \\
\hline Methotrexate & $21(77.8)$ \\
\hline Steroid (methylprednisolone) & $23(85.2)$ \\
\hline Cyclophosphamide & $2(7.4)$ \\
\hline Mycophonelate mofetil & - \\
\hline Azathioprine & $1(3.7)$ \\
\hline Calcium Channel Blocker (CCB) & $27(100)$ \\
\hline PPI/H2 Antagonist & $17(63)$ \\
\hline Aspilet & $24(88.9)$ \\
\hline Never receive any treatment before & $3(11.1)$ \\
\hline \multicolumn{2}{|l|}{ Laboratory results, mean $\pm S D$} \\
\hline Hemoglobin (gr/dl) & $12.6 \pm 1.5$ \\
\hline Leukocyte $\left(/ \mathrm{mm}^{3}\right)$ & $9,500 \pm 3,500$ \\
\hline Thrombocyte $\left(/ \mathrm{mm}^{3}\right)$ & $327,000 \pm 86,000$ \\
\hline Creatinin (mg/dl) & $0.66 \pm 0.19$ \\
\hline SGPT (U/L) & $22 \pm 6$ \\
\hline Krebs von den Lungen- 6 (ng/ml), median (range) & $2.3(0.5-8.1)$ \\
\hline \multicolumn{2}{|l|}{ Clinical manifestation based on ACR/ EULAR, n (\%) } \\
\hline Raynaud's phenomenon & $27(100)$ \\
\hline Fibrosis of both arms & $27(100)$ \\
\hline Sclerodactyly & $25(92.6)$ \\
\hline Puffy fingers & - \\
\hline Telangiectasis & $20(74.1)$ \\
\hline Scar of fingers & $15(55.6)$ \\
\hline Calcinosis & - \\
\hline mRSS, median (range) & $23(5-45)$ \\
\hline $\mathrm{FVC}(\%)$, mean $\pm \mathrm{SD}$ & $52 \pm 11$ \\
\hline \multicolumn{2}{|l|}{ Restrictive lung disorder, $\mathrm{n}(\%)$} \\
\hline Mild to moderate (FVC 60-79\%) & 7 (25.9) \\
\hline Severe (FVC $\leq 59 \%)$ & $20(74.1)$ \\
\hline
\end{tabular}

\section{Table 2. Correlation between KL-6 serum and FVC value}

\begin{tabular}{lcc}
\hline \multicolumn{2}{c}{ Variable } & \multicolumn{2}{c}{ FVC value } \\
& Correlation coefficient $(\mathrm{r})$ & $p$-value \\
\hline KL-6 serum level & $-0,118$ & $0,279^{*}$ \\
\hline
\end{tabular}

Notes: Krebs von den Lungen-6 (KL-6), forced vital capacity (FVC)

*significant if $\mathrm{p}$-value $<0.05$ 




Figure 1. Scattered diagram of KL-6 level and FVC value

Notes: Krebs von den Lungen-6 (KL-6), forced vital capacity (FVC)

\section{Table 3. Correlation between KL-6 serum and mRSS}

Variable

KL-6 serum level

Notes: modified Rodnan skin score (mRSS), forced vital capacity (FVC)

*significant if $\mathrm{p}$-value $<0.05$
mRSS

Correlation coefficient $(\mathrm{r})$ p-value

0,312 * 


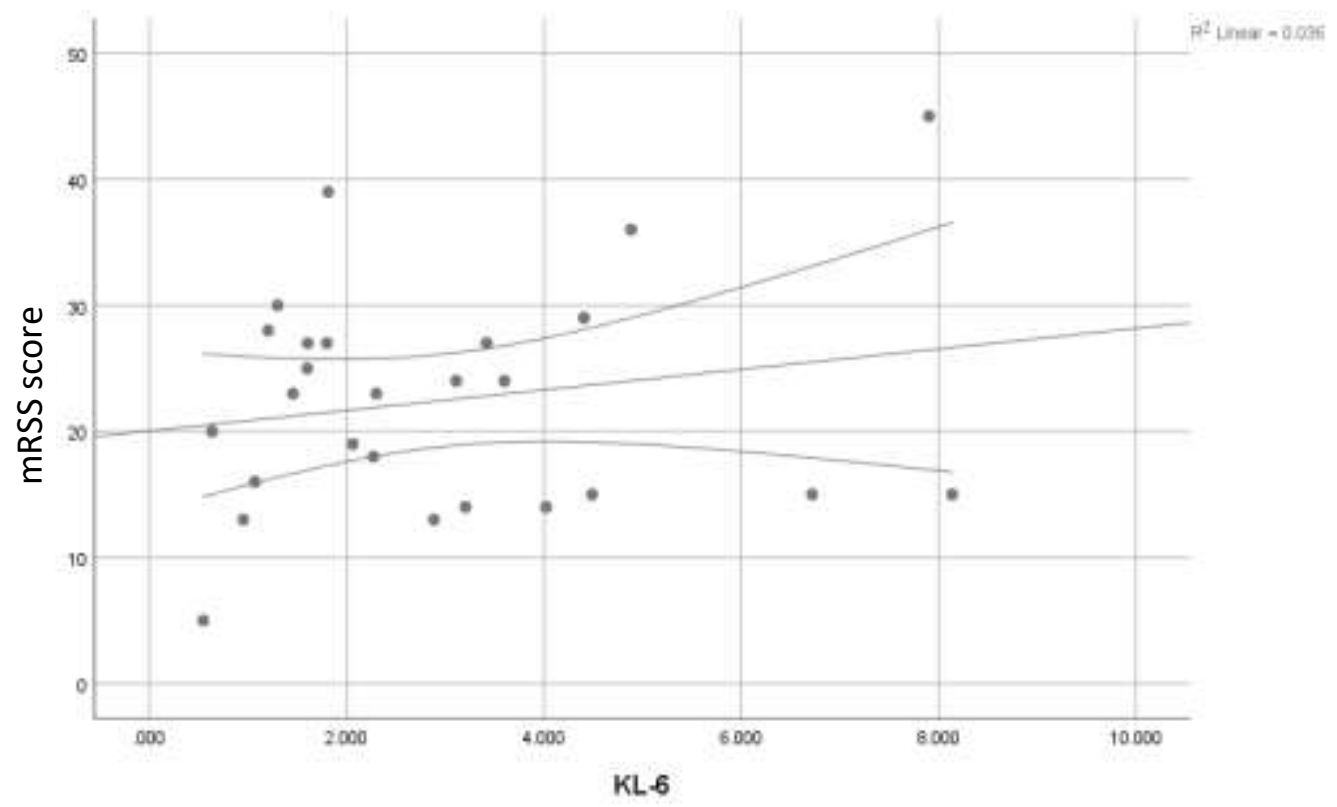

\section{Figure 2. Scattered diagram of KL-6 level and mRSS}

*significant if $\mathrm{p}$-value $<0.05$

\section{Discussion}

\section{Baseline characteristics}

In this study, the mean age of SSc patients was 42 years old. This was echoed the findings in the other studies which stated that the highest SSc onset was on the third until fourth decade of life. ${ }^{14}$ A study in Indonesia conducted by Salim et al shown that the average age of SSc patients was $41 \pm 11$ years. ${ }^{9}$ Similar findings were also reported in a cohort study by Sujau et al from Malaysia which involved $90.3 \%$ female subjects with the mean age of 42.6 years old. ${ }^{15}$ Another study conducted in Singapore by Low et al reported mean age of $46 \pm 14.9$ years in their subjects. ${ }^{16}$

Range of mRSS value in this study was 5-45 with the mean score of 23. It was in accordance with a cohort study from Egypt which showed mRSS value ranged from 4 until 45. ${ }^{17}$ Mean FVC value of the subjects was $52 \%$. It was quite consistent with the findings from Salim et al study which stated FVC value of $58.2 \pm 10.8 \% .9$ Classification of restrictive lung disorder based on FVC value in this study were mostly severe restrictive lung disorder $(74.1 \%)$, whilst the rest of the subjects $(25.9 \%)$ had mild to moderate restrictive lung disorder. This was in accordance with study by Steen et al which shown moderate to severe restrictive lung disorder in $40 \%$ of subjects. ${ }^{18}$

KL-6 serum with FVC value
This study was the first study in Indonesia to analyze the correlation between KL-6 serum with FVC value and mRSS. In this study, there was no correlation between KL- 6 serum and FVC value. It was different with another study from Benyamine et al and Bonela et al which showed a significant negative correlation between KL-6 serum and FVC value. ${ }^{19,20}$ Benyamin et al reported in their study that KL- 6 serum significantly correlated with FVC value $(\mathrm{r}=-0.418 ; p=0.001)$ on SSc patients in France. ${ }^{20}$ Study in Italy by Bonela et al also showed a significant negative correlation between KL- 6 serum and FVC value $(r=-0.47, p<0.05) .{ }^{19}$ Both Benyamin et al and Bonela et al used HRCT and PFT to assess pulmonary fibrosis on SSc patients.

The difference on KL-6 serum with FVC value between this study and previous studies might happen due to several things. First, the subjects of this study have different degree of skin thickening on chest area. This altered the ability of chest cavity to expand, hence disrupting accuracy of spirometry test. The decrease in FVC value due to lung fibrosis is the one that was supposed to be measured. In addition to that, the mean age of subjects in this study was 42 years old, in which their volume of lungs might had already decreased. Secondly, KL-6 serum in this study was measured using ELISA method instead of chemiluminescent enzyme immunoassay (CLEIA) method which was used in most 
of KL-6 studies in the other countries. However, in Indonesia, CLEIA method was not possible to be performed on daily clinical practice.

\section{$K L-6$ serum with $m R S S$}

This study also showed no significant correlation between KL-6 serum and mRSS. This was consistent with cohort study from Cao et al in China who reported lack of correlation between KL- 6 serum and mRSS score in 141 SSc patients (60 dSSc patients and 81 1SSc). ${ }^{21}$ This lack of correlation was also reported in Hant et al study, named Scleroderma Lung Study (SLS), in North America. ${ }^{22}$ Benyamin et al also reported that there was no significant level of KL-6 serum in dSSc patients compared to 1 SSc. 20

On the other hand, Bonella et al reported different results. They reported that there was a significant correlation between KL- 6 and mRSS score ( $r=0.71$, $\mathrm{p}<0.0001) .{ }^{19}$ However, they include 10 1SSc and only 15 dSSc patients, a fewer amount of subjects compared to the other studies.

We could not differentiate whether the restrictive lung disorder was caused by lung fibrosis or caused by skin fibrosis on chest area, which might affect the results of this study. The measurement of KL- 6 serum in this study used KL-6 / Human MUC-1, in which has not been used in any published study and might need some calibrations.

\section{Conclusion}

There was no correlation between serum KL-6 levels with FVC and mRSS value of patient with restritive lung disease in diffuse type systemic sclerosis. Further study analyzing KL-6 serum with lung fibrosis in SSc patients, diffuse and limited type, using HRCT is warranted.

\section{References}

1. Allanore $\mathrm{Y}$, Simms $\mathrm{R}$, Oliver Distler $\mathrm{O}$, Trojanowska M, Pope J, Denton CP, et al. Systemic sclerosis. Nat Rev Dis Primers. 2015;(1): 1-21.

2. Hassoun PM. Lung involvement in systemic sclerosis. Presse Med. 2011;40(1):e25-39.

3. Jain S, Shahane A, Derk CT. Interstitial Lung Disease in Systemic Sclerosis: Pathophysiology, Current and New Advances in Therapy. Inflamm Allergy Drug Targets. 2012;(11): 266-77.

4. Bauer PR, Schiavo DN, Osborn TG, Levin DL, Sauver JS, Hanson AC, et al. Influence of interstitial lung disease on outcome in systemic sclerosis: a population-based historical cohort study. Chest. 2013;144(2):571-7.

5. D'Angelo WA, Fries JF, Masi AT, Shulman LE. Pathologic observations in systemic sclerosis (scleroderma): a study of fifty-eight autopsy cases and fifty-eight matched controls. The American journal of medicine. 1969;46(3):42840.
6. Varga, J. Systemic sclerosis: an update. Bull NYU Hosp Jt Dis. 2008; 66(3): 198-202.

7. Solomon JJ, Olson AL, Fischer A, Bull T, Brown KK, Raghu G. Scleroderma lung disease. Eur Respir Rev. 2013;22(127):6-19.

8. Mango RL, Ryu JH, Makol A. Newer insights into the management of interstitial lung disease in systemic sclerosis. Indian $\mathrm{J}$ Rheumatol. 2017(12):S194-203.

9. Salim S, Wachjudi RG, Dewi S. Correlation of sCD40L Level with Force Vital Capacity Value in Restrictive Lung Disease of Systemic Sclerosis Patients. Indonesian Journal of Rheumatology 2018;10(1):20-23.

10. Kumanovics G, Pentek M, Bae S, Daniela D, Khanna D, Furst DE, et al. Assessment of skin involvement in systemic Sclerosis. Rheumatology. 2017;56:53-66.

11. Wu W, Jordan S, Graf N, de Oliveira PJ, Curram $\mathrm{J}$, Allanore Y, et al. Progressive skin fibrosis is associated with a decline in lung function and worse survival in patients with diffuse cutaneous systemic sclerosis in the European Scleroderma Trials and Research (EUSTAR) cohort. Ann Rheum Dis 2019;0:1-9.

12. Lota HK, Renzoni EA. Circulating Biomarkers of Interstitial Lung Disease in Systemic Sclerosis. The Journal of Rheumatology. 2012;39(6):11201124.

13. Horimasu Y, Hattori N, Ishikawa N, Kawase S, Tanaka S. Utility of KL-6/MUC1 in the clinical management of interstitial lung diseases Respiratory Medicine. 2012;106: 1756e-1764.

14. Varga J. Systemic sclerosis; epidemiology, pathology and pathogenesis. Primer on rheumatic disease 13 th ed Atlanta: Springer.2008:351-8.

15. Sujau I, Ng CT, Sthaneshwar P, Sockalingam S, Cheah TE, Yahya $F$, et al. Clinical and autoantibody profile in systemic sclerosis: baseline characteristics from a West Malaysian cohort. Int J Rheum Dis 2015;18(4):459-65.

16. Low A, Gee TG, Giap LW, Cheng NS, Santosa A, Chan G, et al. Disease Characteristics of the Singapore Systemic Sclerosis Cohort. Proceedings of Singapore Healthcare. 2013;22(1):8-14.

17. Mahmoud A, Alhefny A, Abugabal M, Abdelmoteleb S, Alhassanein KF, Abdelzaher A et al. Characteristics of Progressive Systemic Sclerosis in a Cohort of Egyptian Patients. Arch Med. 2018;10:1-7.

18. Steen VD, Conte C, Owens GR, Medsger Jr TA. Severe restrictive lung disease in systemic sclerosis. Arthritis Rheum. 1994 Aug;37(9):1283-9.

19. Bonella F, Volpe A, Caramaschi P, Nava C, Ferrari P, Schenk K, et al. Surfactant protein D and KL-6 serum levels in systemic sclerosis: correlation with lung and systemic involvement. Sarcoidosis Vasc Diffuse Lung Dis. 2011; 28(1):27-33.

20. Benyamine A, Heim X, Resseguier N, Bertin D, Gomez C, Ebbo M, et al. Elevated serum Krebs 
von den Lungen-6 in systemic sclerosis: a marker of lung fibrosis and severity of the disease. Rheumatol Int. 2018;38(5):813-819.

21. Cao XY, Hu SS, Xu D, Li MT, Wang Q, Hou Y, Zeng XF. Serum levels of Krebs von den Lungen6 as a promising marker for predicting occurrence and deterioration of systemic sclerosis-associated interstitial lung disease from a Chinese cohort. Int $\mathrm{J}$ Rheum Dis. 2018;1-8.

22. Hant FN, Bradley AL, Wang HJ, Li N, Elasshof $\mathrm{R}$, Tashkin DP, et al. Surfactant protein D and KL-6 as serum biomarkers of interstitial lung disease in patients with scleroderma. Journal of Rheumatology. 2009;36(4):773-780. 